\title{
Observations of Hall Reconnection Physics Far Downstream of the $X$ Line
}

\author{
R. Mistry, ${ }^{1}$ J. P. Eastwood, ${ }^{1}$ C. C. Haggerty, ${ }^{2}$ M. A. Shay, ${ }^{2}$ T. D. Phan, ${ }^{3}$ H. Hietala, ${ }^{4}$ and P. A. Cassak ${ }^{5}$ \\ ${ }^{1}$ The Blackett Laboratory, Imperial College London, London SW7 2AZ, United Kingdom \\ ${ }^{2}$ Bartol Research Institute, Department of Physics and Astronomy, University of Delaware, Newark, Delaware 19716, USA \\ ${ }^{3}$ Space Sciences Laboratory, University of California, Berkeley, California 94720, USA \\ ${ }^{4}$ Department of Earth, Planetary and Space Sciences, University of California, Los Angeles, California 90095, USA \\ ${ }^{5}$ Department of Physics and Astronomy, West Virginia University, Morgantown, West Virginia 26506, USA \\ (Received 27 April 2016; revised manuscript received 10 August 2016; published 28 October 2016)
}

\begin{abstract}
Observations made using the Wind spacecraft of Hall magnetic fields in solar wind reconnection exhausts are presented. These observations are consistent with the generation of Hall fields by a narrow ion inertial scale current layer near the separatrix, which is confirmed with an appropriately scaled particle-incell simulation that shows excellent agreement with observations. The Hall fields are observed thousands of ion inertial lengths downstream from the reconnection $X$ line, indicating that narrow regions of kinetic dynamics can persist extremely far downstream.
\end{abstract}

DOI: 10.1103/PhysRevLett.117.185102

Magnetic reconnection plays a crucial role in many plasma systems by reconfiguring the magnetic field topology, heating the plasma, and forming jets of fast plasma outflow [1]. Of key importance for collisionless reconnection is the diffusion region, where kinetic effects result in the topological field change. Within this region electrons and ions decouple from the field on different spatial scales, resulting in differential motion between the species and the generation of Hall currents, Hall magnetic fields, and Hall electric fields [2]. Hall magnetic fields in symmetric antiparallel reconnection produce a characteristic quadrupolar Hall field (e.g., Refs. [3,4]), which is often noted as an indicator of proximity to the diffusion region in in situ satellite observations (e.g., Refs. [5-7]). In the more general case of guide field reconnection, the Hall field is distorted by Lorentz forces acting on the electron current in the ion diffusion region [8-10].

The majority of plasma in the exhaust does not pass through the diffusion region and energy conversions primarily occur across the exhaust boundaries or within the exhaust itself. How this happens is determined by the structure of the exhaust. Understanding the exhaust is therefore important in determining how reconnection affects energy flows in the systems in which it occurs. Reconnection exhausts at large distances from the $X$ line are often described in a magnetohydrodynamics (MHD) framework which does not include kinetic physics (e.g., Refs. [11,12]). Despite this, the kinetic process of ions counterstreaming along magnetic field lines has been observed far downstream in the exhaust region in the solar

Published by the American Physical Society under the terms of the Creative Commons Attribution 3.0 License. Further distribution of this work must maintain attribution to the author(s) and the published article's title, journal citation, and DOI. wind [13]. It is therefore important to understand where non-MHD effects are important in the reconnection process, and whether MHD can sufficiently capture the physics of the exhaust region. In the solar wind, reconnection generally forms quasi-two-dimensional exhausts [14] which are comparable to two-dimensional simulations. Solar wind reconnection occurs with a range of guide field strengths [15] and occurs within a large system (the heliosphere), producing extended exhausts in the outflow direction [16,17]. This makes in situ observations ideal for determining the exhaust structure at much larger distances from the $X$ line than can currently be simulated.

Here we present in situ observations of Hall magnetic fields in solar wind exhausts, revealed by bipolar perturbations to the out-of-plane field. These are compared to an appropriately scaled particle-in-cell (PIC) simulation, showing that the Hall fields are generated by kinetic scale electron flows in the exhaust. These signatures are observed thousands of ion inertial lengths downstream of the $X$ line, indicating that these Hall signatures can persist far downstream and well into regimes typically described with MHD.

Figure 1 shows data from the Wind spacecraft [18] on 2002-03-13 (event 1) in a LMN coordinate system determined by hybrid minimum variance analysis of magnetic field data [19]. $\mathbf{L}$ is the exhaust outflow direction, $\mathbf{M}$ is the out-of-plane direction, and $\mathbf{N}$ is the current sheet normal. The rotation in $B_{L}$ at $01: 53: 46-01: 53: 58$ UT indicates the passage of a current sheet which is accompanied by an exhaust outflow in the $+L$ direction, [panel (f)]. The outof-plane component of the field $\left(B_{M}\right)$ is 1.3 times the reconnecting field $\left(B_{L}\right)$ in the inflow region. $B_{M}$ is increased within the majority of the exhaust (shown in red). One-dimensional Riemann problems [20,21] and twodimensional hybrid simulations [22] of guide field reconnection show that rotational discontinuities form at the 

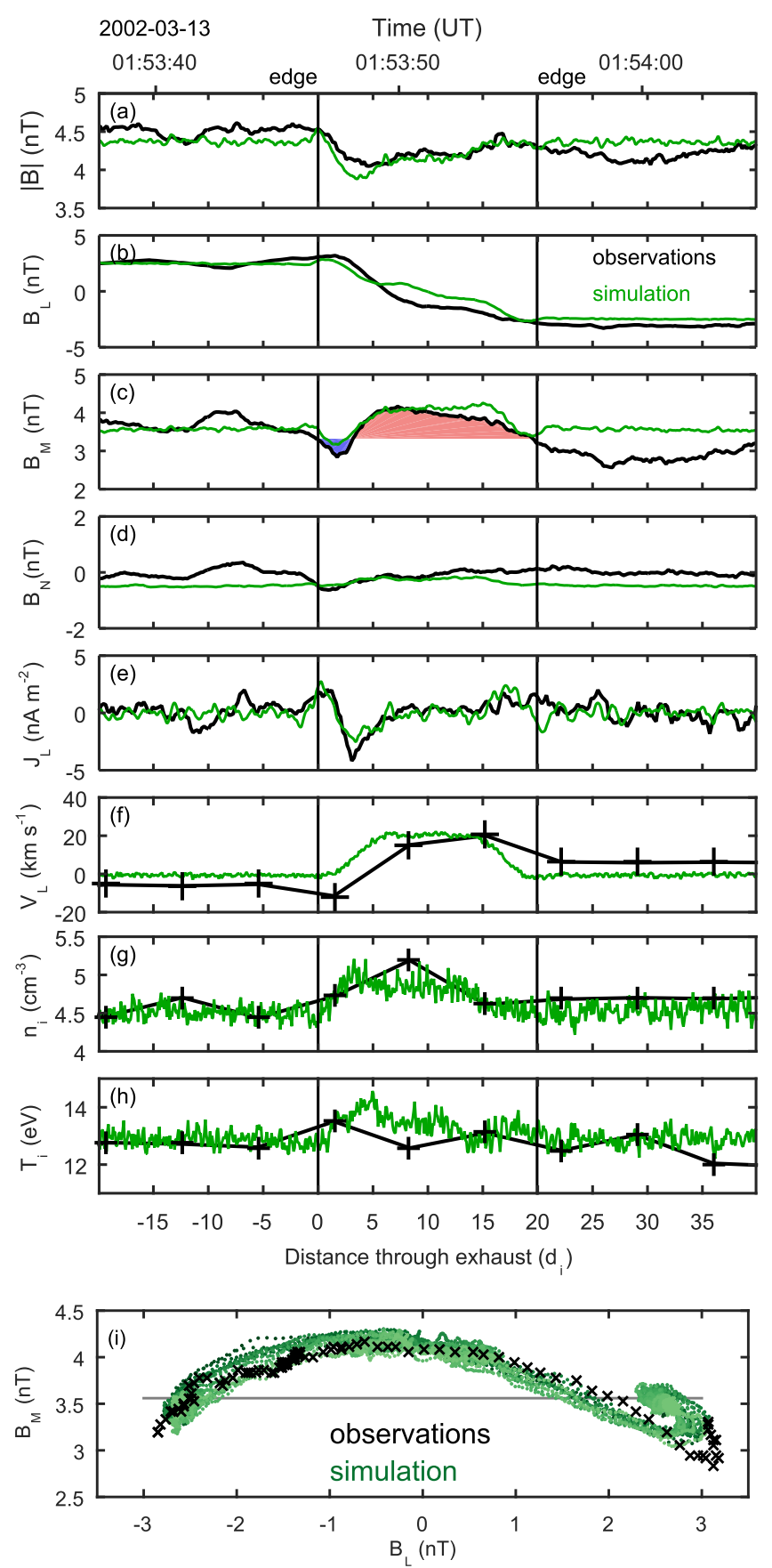

FIG. 1. Observations from Wind in black, as a function of distance through the exhaust in the normal direction $\left(V_{L}\right.$ has been shifted by $274 \mathrm{~km} \mathrm{~s}^{-1}$ ). (a-d) Magnetic field. (e) L-component of current. (f) L-component of ion velocity. (g) Ion density. (h) Ion temperature. Times of measurements are indicated at the top. Vertical lines indicate the exhaust edges. Simulation data from a cut at $L=80 d_{i}$ are shown in green in physical units. (i) Magnetic field hodograms from cuts [shown in Fig. 2(b)] at $25 \leq L \leq$ $110 d_{i}$ are compared to Wind observations.

exhaust boundaries which rotate the magnetic field from the reconnection plane to the out-of-plane direction, resulting in a unipolar increase of $B_{M}$ within the exhaust. In addition to the observed $B_{M}$ increase, $B_{M}$ decreases at the beginning of
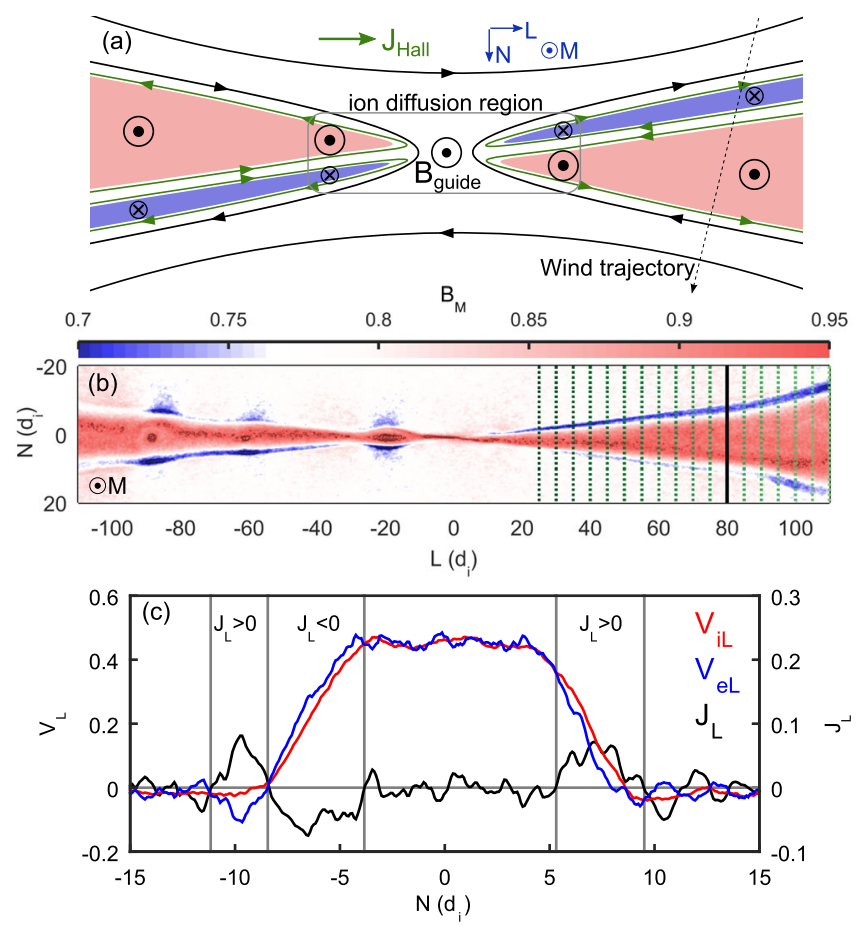

FIG. 2. (a) Sketch of the reconnection exhaust encounter. Positive and negative Hall magnetic fields are shown in red and blue, respectively. (b) Out-of-plane magnetic field from the simulation. $L=80 d_{i}$ is shown in black. (c) $V_{\mathrm{iL}}, V_{\mathrm{eL}}$, and $J_{L}$ at $L=80 d_{i}$. (b) and (c) are in simulation units.

the exhaust at $01: 53: 46.7-01: 53: 48.1$ UT (shown in blue), resulting in a bipolar perturbation to $B_{M}$. Figure 2(a) shows the expected Hall field within the ion diffusion region and Wind's trajectory through the reconnection exhaust. The polarity of the observed bipolar perturbation to $B_{M}$, and that the decrease in $B_{M}$ is smaller in magnitude and duration than the increase, is qualitatively in agreement with previous studies on the distortion of the quadrupole structure in symmetric reconnection $[8,10]$. Spatial distances in the normal direction corresponding to time intervals $(\delta t)$ are measured as $\left(\mathbf{V}_{\mathbf{s w}} \cdot \mathbf{N}\right) \delta t$, where $\mathbf{V}_{\mathbf{s w}}$ is the solar wind velocity. The total exhaust width is measured to be $25 d_{i}$, where $d_{i}$ is the ion inertial length using the mean ion density in the inflow regions $\left(4.7 \mathrm{~cm}^{-3}\right)$.

A quantitative comparison is made between these observations and a fully kinetic 2.5-dimensional PIC simulation using the P3D code [23]. Magnetic field strengths and particle number densities are normalized to their values in the inflow regions, $\left|B_{0}\right|$ and $n_{0}$, respectively. Lengths are normalized to the ion inertial length $d_{i}=c / \omega_{p i 0}$ at $n_{0}$, and velocities to the Alfvén speed $c_{A 0}=\sqrt{ }\left[B_{0}^{2} /\left(\mu_{0} m_{i} n_{0}\right)\right]$ (the speed of light is $\left.c / c_{A 0}=11\right)$. Simulations are performed in a periodic domain with a system size of $S_{L} \times S_{N}=$ $366.4 d_{i} \times 183.2 d_{i}$, with 100 particles per grid in the inflow region. The system was initialized with two Harris current sheets superimposed on an ambient population (as detailed in Ref. [24]), with an ion-to-electron mass ratio of 25 . The 
guide field is 1.3 times the reconnecting field, and ion and electron beta are 1.2 and 1.7 , respectively, to match observations in the inflow regions.

Figure 2(b) shows the simulation out-of-plane magnetic field at a time when reconnection reached a steady state (57 ion cyclotron periods), in the same coordinate system used for observations. The simulation exhaust width at $L=80 d_{i}$ is comparable to the observed exhaust width. Simulation data from this cut are compared to observed data in Fig. 1 by converting from simulation units to physical units. The shear in $V_{L}$ across the exhaust in the observations is not included in the simulation. Despite this we find excellent agreement between the simulated and observed data, including $B_{M}$. The width of the exhaust increases with distance from the $X$ line, making it difficult to compare other spatial cuts to the observations. We therefore compare magnetic field hodograms from cuts at $25 \leq L \leq 110 d_{i}$ at $5 d_{i}$ intervals to the observed data in Fig. 1(i). There is excellent agreement in magnetic structure at all cut locations.

The $B_{M}$ Hall field is associated with a $J_{L}$ Hall current. $L$ components of the current, ion $\left(V_{\mathrm{iL}}\right)$ and electron $\left(V_{\mathrm{eL}}\right)$ velocities at $L=80 d_{i}$ are shown in Fig. 2(c). $V_{\text {iL }}$ is positive throughout the exhaust. $V_{\text {eL }}$, however, is negative at $-11<N<-9$. Further analysis shows that here $V_{\mathrm{eL}}$ is largely field aligned, corresponding to electron flow towards the $X$ line. $J_{L}$ is positive, which decreases $B_{M}$ and generates the negative Hall field. Similar electron flows can also be seen closer to the $X$ line in smaller-scale simulations [25].

At $-8<N<9$, the electron velocity is shifted in the $-N$ direction relative to the ion velocity. At $-8<N<-4$, $V_{\text {iL }}<V_{\text {eL }}$ and $J_{L}$ is negative. At $5<N<8, V_{\text {iL }}>V_{\mathrm{eL}}$ and $J_{L}$ is positive. The Hall magnetic fields are therefore generated by current systems [shown in Fig. 2(a)] associated with an electron flow towards the $X$ line at one separatrix, as well as a relative shift between the ion and electron outflows in the $N$ direction. Note that this shift is qualitatively consistent with the deflection of the in-plane electron current in the ion diffusion region in the presence of a guide field (and is associated with the kinetic Alfvén wave structure of guide field reconnection [26]), and that the negative $J_{L}$ current remains significantly deflected from the midplane far downstream of the $X$ line. Wind ion and electron velocities cannot be resolved at sufficiently high resolution to directly determine $J_{L}$; therefore, $J_{L}$ is calculated from Wind magnetic field observations, $J_{L} \approx$ $-d\left(B_{M}\right) / d N$ [the contribution from $d\left(B_{N}\right) / d(M)$ is negligible as $B_{N} \sim 0$ ], which shows good agreement with the simulation [Fig. 1(e)]. The $J_{L}$ current layer between the negative and positive Hall fields is measured to have a full width at half maximum of $1.1 d_{i}$ and $1.3 d_{i}$ in the observations and simulations, respectively. The presence of these signatures far downstream of the $X$ line indicates that MHD treatments may be unable to sufficiently describe the exhaust region and its structure.
We identify a total of 8 events (including event 1 ) which show clear evidence of Hall magnetic fields from a list of 197 solar wind reconnection exhausts observed by Wind by Ref. [15]. An LMN coordinate system was first determined for each event. Identified events were found by a search for clear bipolar perturbations of $B_{M}$ within the exhaust interval. Positive and negative perturbations to $B_{M}$ were not required to be equal in duration or magnitude as this does not occur in general for guide field reconnection, as illustrated by event 1 and the simulation. Events with strong fluctuations in $B_{N}$ or large differences in $V_{L}$ across the current sheet (more than $75 \%$ of the exhaust outflow speed) were not considered. No requirement was imposed on the polarity of bipolar $B_{M}$ perturbations. The polarity for all 8 identified events was consistent with that expected for Hall fields; no events were identified with the incorrect polarity. Guide fields for the identified events are between 0.3 and 1.6, whereas the range for all events in the list is $0-10$.

Figure 3 shows $B_{M}$ for all 8 events as a function of distance through the exhaust in $N$. At all events the decrease in $B_{M}$ is narrow and confined to the edge of the exhaust. Relative distances from the $X$ line (determined from measurements of the exhaust width and assuming an equal reconnection rate at all events) are shown on the right, indicating that this pattern is observed even at very large distances downstream. From full width at half maximum measurements of $J_{L}$, the current layers between the negative and positive Hall fields are determined to have widths in the normal direction of $0.8-6.0 d_{i}$. In addition to these ion-scale structures which are not an MHD phenomenon, the majority of the exhaust shows an increase in $B_{M}$ which is a fluid effect related to the structure of the exhaust boundaries [20-22].

In single spacecraft observations the Hall field is observed as a bipolar variation in $B_{M}$. To identify the full quadrupolar structure both exhausts must be observed (e.g., Ref. [27]). This also allows for a more accurate determination of each spacecraft's distance from the $X$ line [17]. Reference [16] has previously studied event 7 with complimentary measurements from ACE. The two spacecraft were located on opposite sides of the $X$ line, observed oppositely directed jets, and Ref. [16] determined Wind to be $\sim 9000 d_{i}$ downstream of the $X$ line. Spacecraft measurements are overlaid in Fig. 4 in the coordinate system defined in the above analysis of Wind data. The passage of a current sheet is detected at Wind (ACE) at $09: 25: 36-09: 28: 47$ UT $(08: 24: 24-08: 26: 54$ UT), and is accompanied by an outflow in the $+L(-L)$ direction.

Having identified bipolar changes in $B_{M}$ in Wind data, we carefully reexamined ACE data. $B_{M}$ is increased within the exhaust of both spacecraft; however, at 09:25: $37.7-09: 28: 41.2$ UT and $08: 24: 27.3-08: 24: 33.5 \mathrm{UT}$ it is decreased at Wind and ACE, respectively. These decreases correspond to regions near opposing pairs of separatricies [see Fig. 2(a)]. In guide field reconnection the 

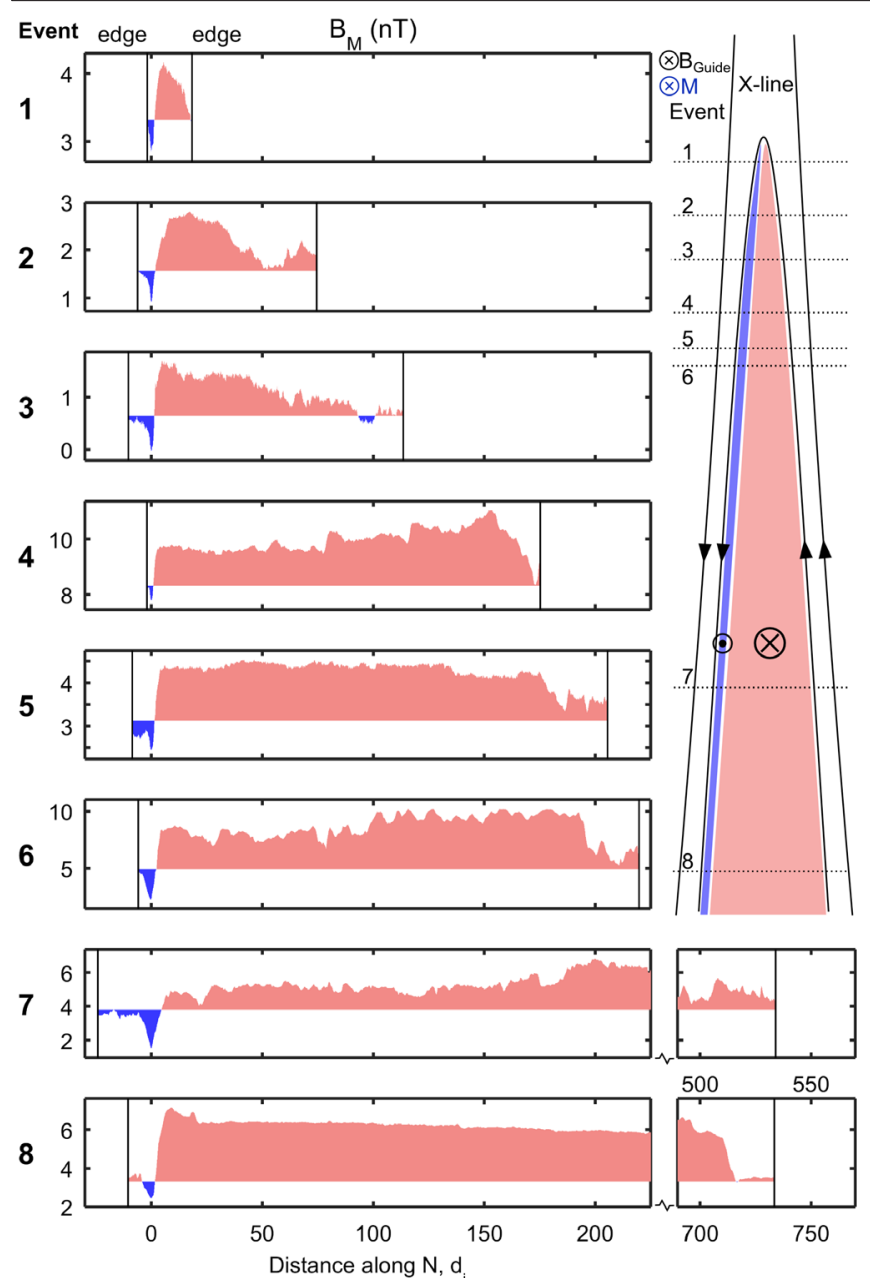

FIG. 3. $B_{M}$ for 8 events, as a function of distance in the normal direction. Positive and negative Hall magnetic field regions are shown in red and blue, respectively. The $x$-axis origin is located at the minima in $B_{M}$. Vertical lines mark the exhausts' edges. Data are shown across two panels for events 7 and 8. Relative distances of observations from the $X$ line are shown on the right.

distortion of the Hall field means that the reversal in the bipolar perturbation in $B_{M}$ does not occur at $B_{L}=0$ (e.g., Refs. [8,28]), as seen in Fig. 1(i). The value of $B_{L}$ at which this does occur differs at the opposing exhausts. Figure 4(f) shows overlaid hodograms of magnetic field data from within the exhaust from both spacecraft. The $B_{L}$ axes have been scaled such that the values of $B_{L}$ at which the bipolar perturbations in $B_{M}$ reverse are coincident and at the center of the figure. The opposite bipolar patterns in Fig. 4(f) reveal the full quadrupolar patterns of the Hall field using both sides of the $X$ line.

In summary, we show, for the first time, clear evidence of the generation of Hall magnetic fields far downstream of the $X$ line in solar wind reconnection exhausts. Additionally, the full quadrupolar structure of the Hall fields is revealed through multispacecraft observations. Although the widths of the exhausts vary between 25
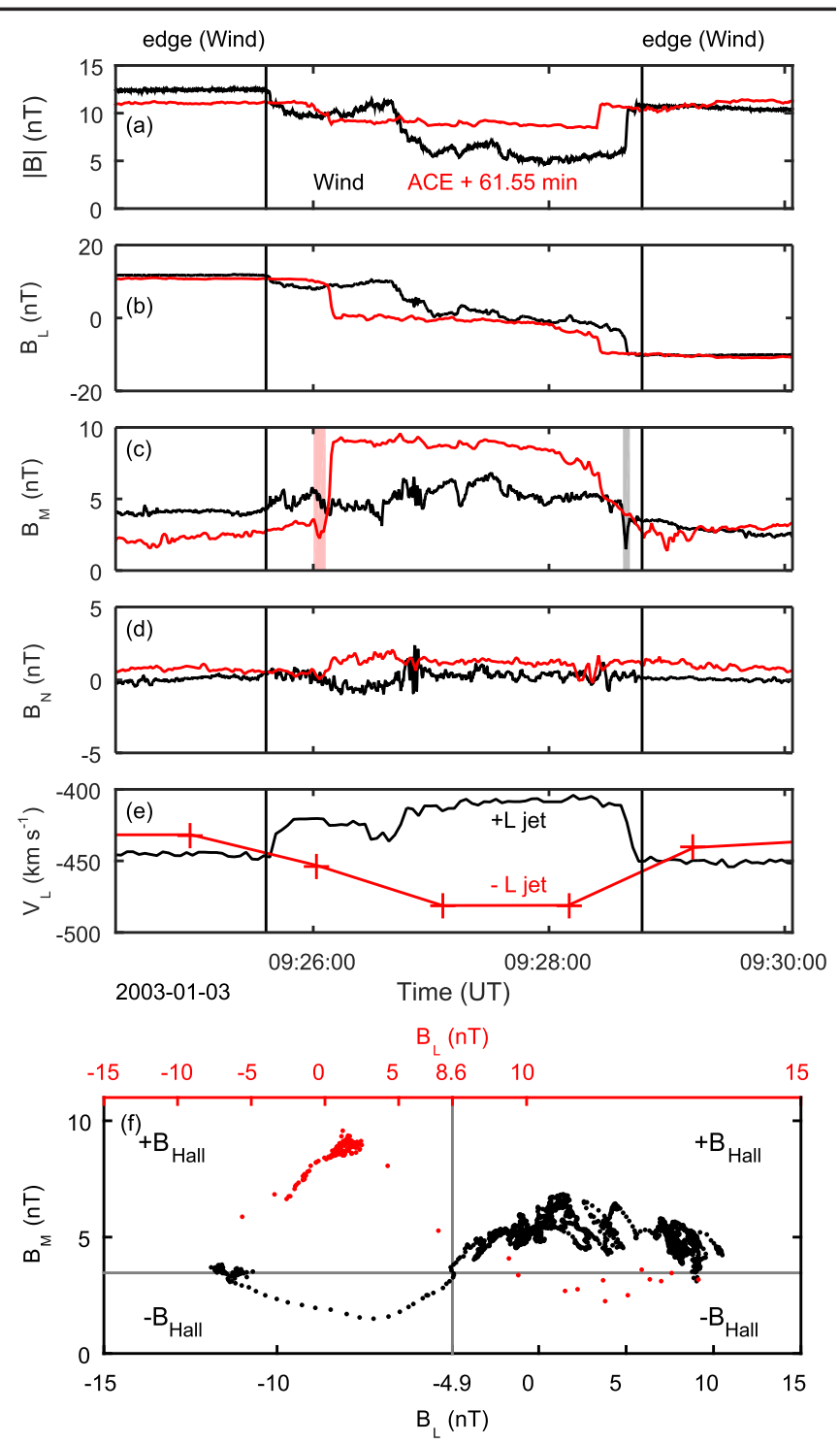

FIG. 4. (a)-(e) Observations from Wind (black) and ACE (red). Vertical lines indicate the exhaust edges at Wind. Shaded regions in (c) indicate intervals of decreased $B_{M}$. (f) Magnetic field hodograms of the exhaust intervals. Wind (ACE) is plotted against the lower (upper) axis. The vertical gray line marks the values of $B_{L}$ at which bipolar perturbations in $B_{M}$ reverse at each spacecraft. The $x$ axes vary linearly on either side of this line. The horizontal line marks the value of $B_{M}$ in the inflow regions at Wind.

and $743 d_{i}$, the width of the current layers associated with the Hall field are narrow $\left(0.8-6.0 d_{i}\right)$. Comparisons to a PIC simulation show that these current layers are supported by thin electron flows towards the $X$ line at the separatricies, and a deflection of the electron flow relative to the ion flow which persists far downstream. We have also performed a two-fluid simulation with the same boundary conditions as used in the PIC simulation. The resulting Hall field does not show good agreement with the observations, as expected given the nature of the Hall field in two-fluid simulations 
[e.g., Ref. [29]]. Hall signatures were clear in only 8 events (guide field of 0.3-1.6). At larger guide fields the decrease in $B_{M}$ is much weaker [30], making the Hall field more difficult to observe [28]. At smaller guide fields fluctuations in the exhaust can obscure Hall fields (although this is more pronounced at lower beta [31]). Furthermore, plasma asymmetries and flow shear between inflow regions can further obscure the presence of Hall signatures, and it is, therefore, perhaps unsurprising that many events from the survey do not show Hall fields despite having guide fields of $0.3-1.6$. A more systematic study is required to identify under exactly which conditions Hall fields are seen.

These observations show that Hall physics can persist thousands of ion inertial lengths downstream of the reconnection $X$ line. Reconnection exhausts can therefore contain localized regions of kinetic dynamics, which may have implications for the interpretation of observational reconnection data and for applications of MHD.

This work was supported by UK STFC through the award of a studentship and Grants No. ST/G00725X/1 and No. ST/N000692/1, NASA Contract No. NAS5-02099, NASA Grants No. NNX15AW58G, No. NNX16AF75G, and No. NNX16AG76G, and NSF Grants No. AGS1219382 and No. AGS-0953463. Simulations and analysis were performed at the National Center for Atmospheric Research Computational and Information System Laboratory (NCAR-CISL) and at the National Energy Research Scientific Computing Center (NERSC). We wish to acknowledge support from the International Space Science Institute and the Turboplasmas project.

[1] M. Yamada, R. Kulsrud, and H. Ji, Rev. Mod. Phys. 82, 603 (2010).

[2] B. Sonnerup, in Solar System Plasma Physics, edited by L. J. Lanzerotti, C. F. Kennel, and E. N. Parker (NorthHolland Publishing Co., Amsterdam, 1979), p. 45.

[3] M. Hesse, J. Birn, and M. Kuznetsova, J. Geophys. Res. 106, 3721 (2001).

[4] M. E. Mandt, R. E. Denton, and J. F. Drake, Geophys. Res. Lett. 21, 73 (1994).

[5] J. P. Eastwood, T. D. Phan, F. S. Mozer, M. A. Shay, M. Fujimoto, A. Retinò, M. Hesse, A. Balogh, E. A. Lucek, and I. Dandouras, J. Geophys. Res. 112, A06235 (2007).
[6] M. Øieroset, T. D. Phan, M. Fujimoto, R. P. Lin, and R. P. Lepping, Nature (London) 412, 414 (2001).

[7] A. Runov et al., Geophys. Res. Lett. 30, 1579 (2003).

[8] J. P. Eastwood, M. A. Shay, T. D. Phan, and M. Øieroset, Phys. Rev. Lett. 104, 205001 (2010).

[9] R. Horiuchi and T. Sato, Phys. Plasmas 4, 277 (1997).

[10] J. D. Huba, Phys. Plasmas 12, 012322 (2005).

[11] H. Petschek, NASA Spec. Publ. 50, 425 (1964).

[12] V. M. Vasyliunas, Rev. Geophys. 13, 303 (1975).

[13] J. T. Gosling, R. M. Skoug, D. J. McComas, and C. W. Smith, J. Geophys. Res. 110, A01107 (2005).

[14] T. D. Phan, J. T. Gosling, and M. S. Davis, Geophys. Res. Lett. 36, L09108 (2009).

[15] T. D. Phan, J. T. Gosling, G. Paschmann, C. Pasma, J. F. Drake, M. Øieroset, D. Larson, R. P. Lin, and M. S. Davis, Astrophys. J. Lett. 719, L199 (2010).

[16] M. S. Davis, T. D. Phan, J. T. Gosling, and R. M. Skoug, Geophys. Res. Lett. 33, L19102 (2006).

[17] R. Mistry, J. P. Eastwood, T. D. Phan, and H. Hietala, Geophys. Res. Lett. 42, 10,513 (2015).

[18] M. H. Acuña, K. W. Ogilvie, D. N. Baker, S. A. Curtis, D. H. Fairfield, and W.H. Mish, Space Sci. Rev. 71, 5 (1995).

[19] J. T. Gosling and T. D. Phan, Astrophys. J. Lett. 763, L39 (2013).

[20] Y. Lin and L. C. Lee, Space Sci. Rev. 65, 59 (1993).

[21] Y. Lin and L.C. Lee, J. Geophys. Res. 100, 19227 (1995).

[22] Y. Lin and D. W. Swift, J. Geophys. Res. 101, 19859 (1996).

[23] A. Zeiler, D. Biskamp, J. F. Drake, B. N. Rogers, M. A. Shay, and M. Scholer, J. Geophys. Res. 107, 1230 (2002).

[24] M. A. Shay, J. F. Drake, and M. Swisdak, Phys. Rev. Lett. 99, 155002 (2007).

[25] P. L. Pritchett, J. Geophys. Res. 106, 3783 (2001).

[26] R. G. Kleva, J. F. Drake, and F. L. Waelbroeck, Phys. Plasmas 2, 23 (1995).

[27] J. P. Eastwood, T. D. Phan, M. Øieroset, and M. A. Shay, J. Geophys. Res. 115, A08215 (2010).

[28] B. N. Rogers, R. E. Denton, and J. F. Drake, J. Geophys. Res. 108, 1111 (2003).

[29] M. A. Shay, J. F. Drake, M. Swisdak, and B. N. Rogers, Phys. Plasmas 11, 2199 (2004).

[30] H. Karimabadi, D. Krauss-Varban, N. Omidi, and H. X. Vu, J. Geophys. Res. 104, 12313 (1999).

[31] K. Higashimori and M. Hoshino, J. Geophys. Res. 120, 1803 (2015). 